PAPER • OPEN ACCESS

\title{
A quaternionic description of kinematics and dynamics universal joint
}

To cite this article: E A Mityushov and N E Misyura 2021 J. Phys.: Conf. Ser. 1901012121

View the article online for updates and enhancements.

The Electrochemical Society

Advancing solid state e electrochenical science \& technology 2021 Virtual Education

Fundamentals of Electrochemistry: Basic Theory and Kinetic Methods Instructed by: Dr. James Noël Sun, Sept 19 \& Mon, Sept 20 at 12h-15h ET

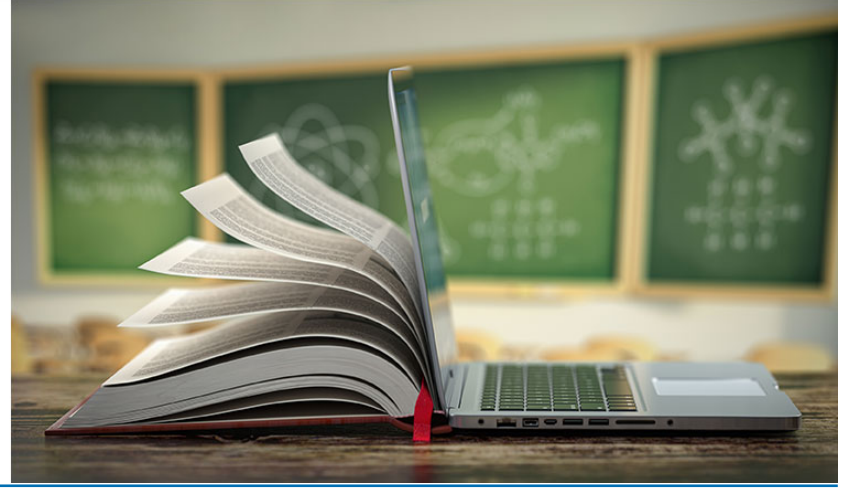




\title{
A quaternionic description of kinematics and dynamics universal joint
}

\author{
E A Mityushov, N E Misyura \\ The First President of Russia B.N. Yeltsyn Ural Federal University, Yekaterinburg, \\ Russia
}

\begin{abstract}
The purpose of the work is figuring out the kinematic and dynamic equations of u-joint motion in the quaternion parametrization. The quaternion description of u-joint kinematics allowed us to formulate the claims about the crosspiece angular velocity projections. We've also determined the principle point projection acting on the crosspiece by solving the inverse problem from Euler's dynamic equations with the found driving shaft's angular velocity and the inertial crosspiece characteristics.

The modeling results are verified in the computer software - Mathcad and can be used for creating optimal u-joint designs by changing its geometry.
\end{abstract}

\section{Introduction}

Cardan-Hooke's joint (or universal joint) - is the main knot of the cardan transmission, which is designed for transferring the rotation between 2 shafts, being at an angle to each other, and consisted of 2 plugs, hard connected with the shafts and with the crosspiece. Picture 1 demonstrates the universal joint with the set of frontal wrenches. The classical results gained from the kinematics study of the u-joint we can see in works [1-5]. The cardan transmissions are widely used in automotive industry, for producing agricultural and и street cars, in mechanical equipment for metal production etc. In the background of massive techno «rearmament» there's a need for creating universal joints with a high carrying capacity, with reduced relative dimensions and increased durability. Dynamic equations in quaternion parameterization that are represented here allow us to gain the link in an analytical form of geometric, kinematic and dynamic characteristic of the u-joint and let us model its movement in different operation modes.

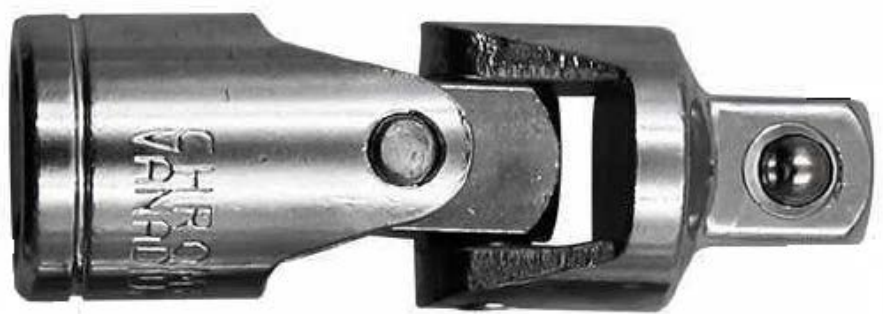

Figure 1. The example of a U-joint 


\section{Known universal joint kinematic relations}

As we see in [5], the link between the angular speed of a driven shaft $\omega_{\text {II }}$ and the angular speed of a main shaft $\omega_{\mathrm{I}}$ in the u-joint is set by the ratio

$$
\omega_{\text {II }}=\frac{\cos \alpha}{1-\sin ^{2} \alpha \sin ^{2} \varphi_{\mathrm{I}}} \omega_{\mathrm{I}},
$$

where $\varphi_{\mathrm{I}}$-is the rotation angle of the main shaft, $\alpha$ - is the angle between shafts' axis.

Traditionally that result was found by the differentiation of an equation, which links the rotation angles of the main shaft $\varphi_{\mathrm{I}}$ and the driven shaft $\varphi_{\mathrm{II}}$, that was found by the geometric method:

$$
\operatorname{tg} \varphi_{\mathrm{II}}=\operatorname{tg} \varphi_{\mathrm{I}} \cos \alpha
$$

Beside the formula (1), that includes that at the initial state the plug of main shaft located perpendicularly to the plane of crossing axis shafts often uses yet another equation:

$$
\omega_{\text {II }}=\frac{\cos \alpha}{1-\sin ^{2} \alpha \cos ^{2} \varphi_{\mathrm{I}}} \omega_{\mathrm{I}}
$$

This formula is figured out for the case when the plug of the main shaft is located in the plane of crossing axis shafts.

The kinematic scheme of the U-joint is shown on figure 2. For forming the crosspiece position of the U-joint the following plane angles are used: $\vartheta$ - the pitch angle, $\psi$ - the destination angle, $\gamma-$ the roll angle (Figure 2). The beginning of a stationary coordinate system is placed in the middle of the crosspiece, axis $O x_{2}$ of the stationary coordinate system is headed vertically. Axis $O x_{1}$ is located in the horizontal plane of the main shaft's plug. The pitch angle $\vartheta$ matches the rotation angle of the main shaft around the axis $O x_{3}$ of the stationary coordinate system. The result of the main shaft spinning determines the coordinate system $O x_{1}{ }^{\prime} X_{2}{ }^{\prime} X_{3}$. While the destination angle $\psi$ is zero. The roll angle $\gamma$ is found as a result of turning the crosspiece around axis $O x_{1}{ }^{\prime}$, which is carried out from axis $O x_{2}{ }^{\prime}$ to axis $O x_{3}{ }^{\prime}$. Consistent executing of those two turns gives us the moving coordinate system $O x_{1}{ }^{\prime \prime} x_{2}{ }^{\prime \prime} x_{3}{ }^{\prime \prime}$ connected with the crosspiece.

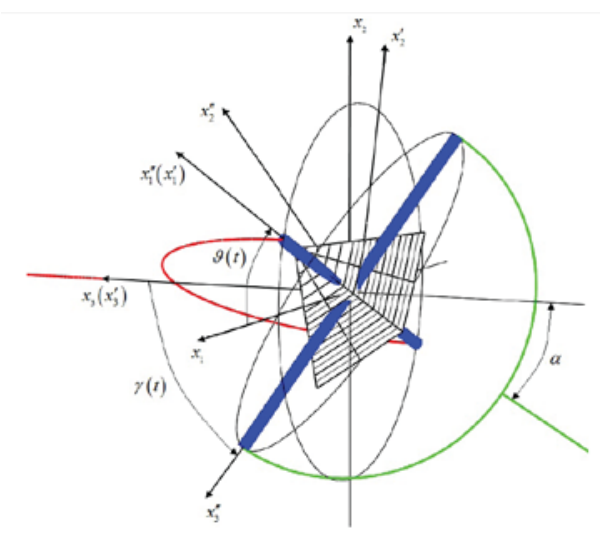

Figure 2. Using plane's angles for describing of u-joint crosspiece position 
The U-joint kinematics mathematically submits to the following main statements.

In every fixed motion moment angles $\vartheta, \gamma$ and $\alpha$, determining the u-joint crosspiece position, are bound by the equations:

$$
\operatorname{tg} \alpha \operatorname{tg} \gamma \operatorname{tg} \vartheta=1
$$

From which follows that when the u-joint crosspiece moves the roll angle $\gamma$ changes according to the law:

$$
\gamma(t)=\frac{\pi}{2}-\operatorname{arctg}(\operatorname{tg} \alpha \cos \vartheta(t)) .
$$

In every fixed moment for angles $\varphi_{\mathrm{I}}, \varphi_{\mathrm{II}}$ and $\alpha$ spins of the main and driven shafts equality holds (1) Considering the range of function values $\operatorname{arctg}\left(\operatorname{tg} \phi_{I} \cos \alpha\right)$, the equation (2) doesn't let us write down the law of changing the rotation angle of the driven shaft on the entire time interval and the law of driven shaft spinning is better find using the equality integration method (1). As a result, we get:

$$
\varphi_{\mathrm{II}}=\int_{0}^{t} \frac{\cos \alpha}{1-\sin ^{2} \alpha \sin ^{2} \varphi_{\mathrm{I}}(t)} \dot{\varphi}_{\mathrm{I}} d t .
$$

\section{The quaternion model of the universal joint kinematics}

These formulated statements let us do the complete description of the u-joint crosspiece spherical movements. To do so we just need to take the advantage of the quaternion model, by which the coordinates of the single orientation quaternion $\mathrm{q}=\left[q_{0}, q_{1}, q_{2}, q_{3}\right], q_{0}^{2}+q_{1}^{2}+q_{2}^{2}+q_{3}^{2}=1$ are linked with the plane angles by the following ratios [6, 7]:

$$
\begin{aligned}
& q_{0}=\cos \frac{\vartheta}{2} \cos \frac{\psi}{2} \cos \frac{\gamma}{2}+\sin \frac{\vartheta}{2} \sin \frac{\psi}{2} \sin \frac{\gamma}{2} \\
& q_{1}=\cos \frac{\vartheta}{2} \cos \frac{\psi}{2} \sin \frac{\gamma}{2}-\sin \frac{\vartheta}{2} \sin \frac{\psi}{2} \cos \frac{\gamma}{2} \\
& q_{2}=\cos \frac{\vartheta}{2} \sin \frac{\psi}{2} \cos \frac{\gamma}{2}+\sin \frac{\vartheta}{2} \cos \frac{\psi}{2} \sin \frac{\gamma}{2} \\
& q_{3}=\sin \frac{\vartheta}{2} \cos \frac{\psi}{2} \cos \frac{\gamma}{2}-\cos \frac{\vartheta}{2} \sin \frac{\psi}{2} \sin \frac{\gamma}{2}
\end{aligned}
$$

In case of even spinning of the main shaft with the angle speed $\omega_{1}$ considering the equations (3), (4) the quaternion coordinate in every fixed time moment can be figured out with such equations:

$$
\begin{aligned}
q_{0} & =\cos \frac{\omega_{I} t}{2} \cos \frac{1}{2}\left[\frac{\pi}{2}-\operatorname{arctg}\left(\operatorname{tg} \alpha \cos \omega_{I} t\right)\right], \\
q_{1} & =\cos \frac{\omega_{I} t}{2} \sin \frac{1}{2}\left[\frac{\pi}{2}-\operatorname{arctg}\left(\operatorname{tg} \alpha \cos \omega_{I} t\right)\right], \\
q_{2} & \left.=\sin \frac{\omega_{I} t}{2} \sin \frac{1}{2}\left[\frac{\pi}{2}-\operatorname{arctg}\left(\operatorname{tg} \alpha \cos \omega_{I} t\right)\right)\right], \\
q_{3} & =\sin \frac{\omega_{I} t}{2} \cos \frac{1}{2}\left[\frac{\pi}{2}-\operatorname{arctg}\left(\operatorname{tg} \alpha \cos \omega_{I} t\right)\right] .
\end{aligned}
$$

While

$$
q_{0}^{2}+q_{1}^{2}=\cos ^{2} \frac{\omega_{I}}{2}, \quad q_{2}^{2}+q_{3}^{2}=\sin ^{2} \frac{\omega_{I}}{2}, \quad q_{0}^{2}+q_{3}^{2}=\cos ^{2} \frac{\gamma}{2}, \quad q_{1}^{2}+q_{2}^{2}=\sin ^{2} \frac{\gamma}{2}
$$
equation:

The position of the crosspiece given its initial state can be found by the following matrix 
where $R(t)=\left(\begin{array}{ccc}q_{0}^{2}+q_{1}^{2}-q_{2}^{2}-q_{3}^{2} & 2\left(q_{1} q_{2}-q_{0} q_{3}\right) & 2\left(q_{0} q_{2}+q_{1} q_{3}\right) \\ 2\left(q_{0} q_{3}+q_{1} q_{2}\right) & q_{0}^{2}-q_{1}^{2}+q_{2}^{2}-q_{3}^{2} & 2\left(q_{2} q_{3}-q_{0} q_{1}\right) \\ 2\left(q_{1} q_{3}-q_{0} q_{2}\right) & 2\left(q_{0} q_{1}+q_{2} q_{3}\right) & q_{0}^{2}-q_{1}^{2}-q_{2}^{2}+q_{3}^{2}\end{array}\right)$ - is the rotation matrix.

In the computer algebra pack on Mathcad with given values $\omega_{1}=0,5 \frac{\mathrm{rad}}{\mathrm{sec}}$ and $\alpha=\frac{\pi}{6}$ we can get the ujoint crosspiece moving animation. The dependences of changing the roll angle, the rotation angle for the driven shaft and also the quaternion coordinate from time are displayed on figure 3,4, and 5 .

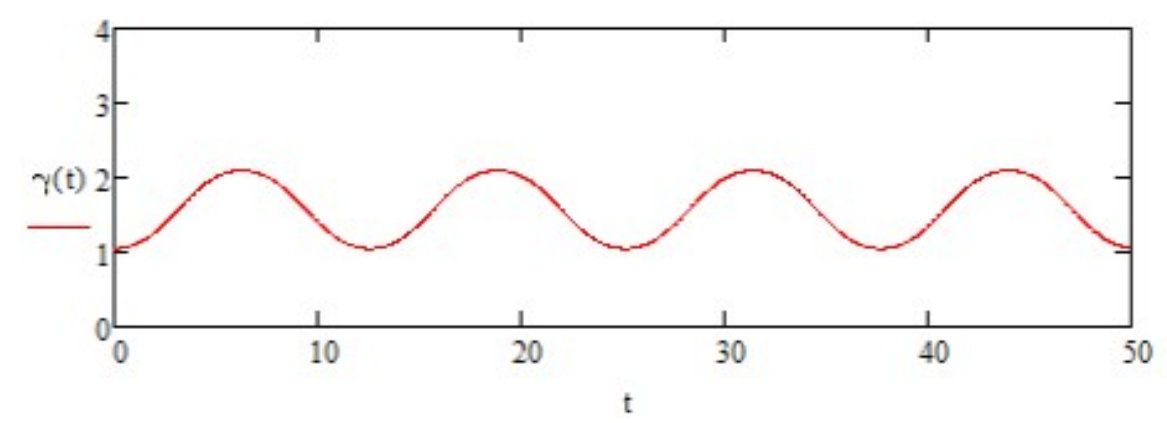

Figure 3. The dependence of changing the roll angle of the u-joint crosspiece from time

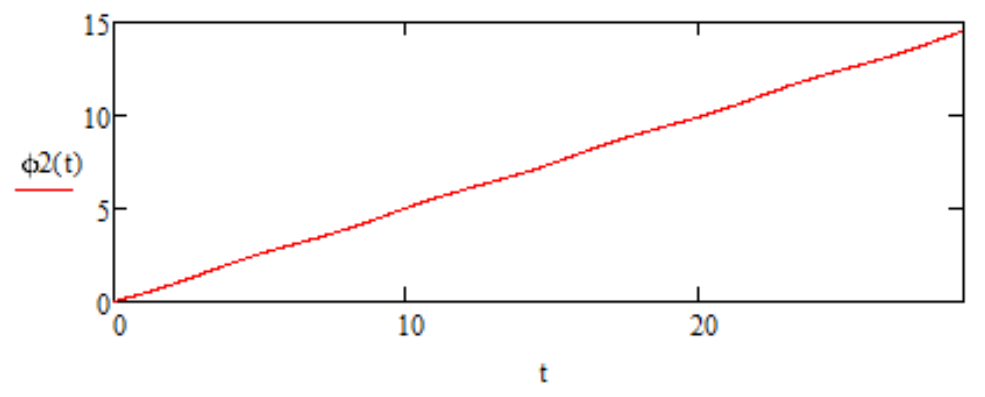

Figure 4. The dependence of changing the rotation angle of the driven shaft from time

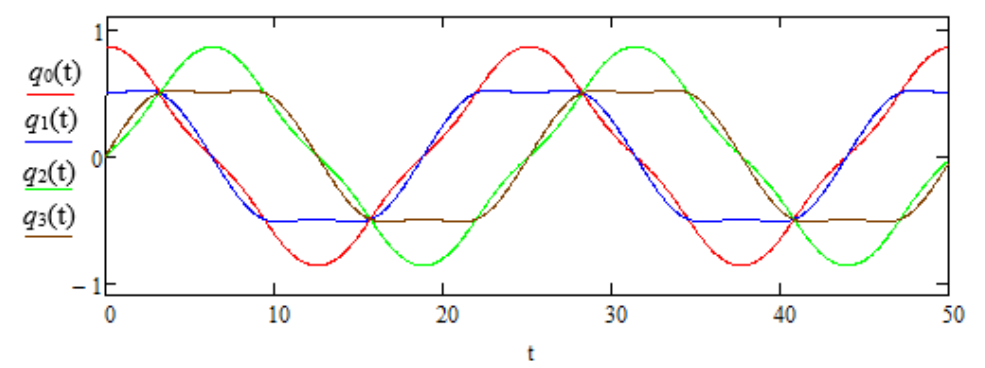

Figure 5. The dependences of changing quaternion orientation coordinates of the u-joint crosspiece from time

The functions of changing quaternion coordinates help us find the projection of the angle speed vector to the connected axis thanks to these kinematic equations [6,7]:

$$
\begin{aligned}
& \Omega_{1}=2\left(\dot{q}_{1} q_{0}-\dot{q}_{0} q_{1}-\dot{q}_{3} q_{2}+\dot{q}_{2} q_{3}\right), \\
& \Omega_{2}=2\left(\dot{q}_{2} q_{0}-\dot{q}_{0} q_{2}-\dot{q}_{1} q_{3}+\dot{q}_{3} q_{1}\right), \\
& \Omega_{3}=2\left(\dot{q}_{3} q_{0}-\dot{q}_{0} q_{3}-\dot{q}_{2} q_{1}+\dot{q}_{1} q_{2}\right) .
\end{aligned}
$$


At the same time the projections of the u-joint crosspiece angle speed to the moving axis go along with the following differential equations:

$$
\dot{\Omega}_{3}+\Omega_{1} \Omega_{2}=0, \dot{\Omega}_{2}-\Omega_{3} \Omega_{1}
$$

Which leads us to when the u-joint crosspiece moves this equation is true:

$$
\Omega_{2}^{2}+\Omega_{3}^{2}=\text { const. }
$$

The quaternionic description of the u-joint kinematics let's us also formulate the following statements relating the projections of the crosspiece angle speed to the moving axis.

In every fixed moment of movement at spinning of the main shaft with the constant angle speed $\omega_{1}$ this condition is satisfied

$$
\Omega_{2}^{2}+\Omega_{3}^{2}=\omega_{1}^{2}
$$

Figure 6 displays the dependences of changing the vector coordinates of the angle speed from time with the given values $\omega_{I}=0,5 \frac{\mathrm{rad}}{\mathrm{sec}}$ and $\alpha=\frac{\pi}{6}$

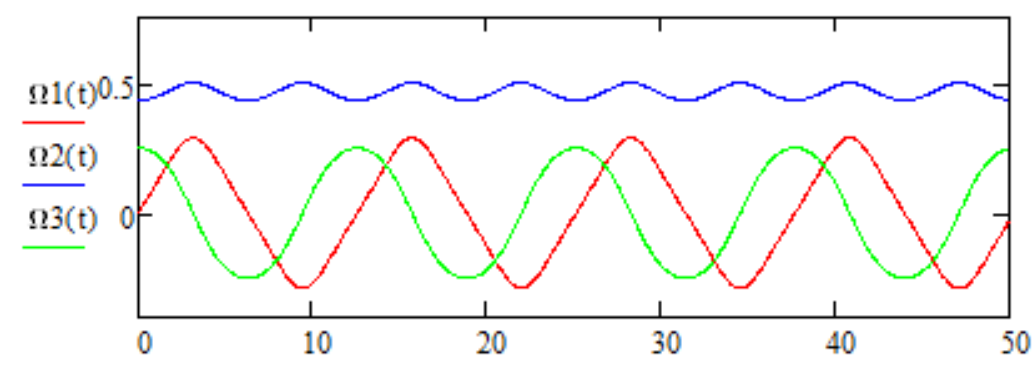

Figure 6. The dependences of changing the vector coordinates of the angle speed from time at the linked axis

The verification of the suggested description for kinematics of the u-joint is made in the computer algebra pack Mathcad [8].

\section{The U-joint crosspiece dynamics}

With the known angle speed of the main shaft and inertial crosspiece characteristics of the main moment of force projection, action on the crosspiece, can be determined by solving the reverse task from Euler's dynamic equations. Let's say the main crosspiece inertial moments $I_{1}, I_{2}$ и $I_{3}$ are set, then we have

$$
\begin{aligned}
& M_{1}=I_{1} \dot{\Omega}_{1}+\Omega_{2} \Omega_{3}\left(I_{3}-I_{2}\right), \\
& M_{2}=I_{2} \dot{\Omega}_{2}+\Omega_{3} \Omega_{1}\left(I_{1}-I_{3}\right), \\
& M_{3}=I_{3} \dot{\Omega}_{3}+\Omega_{1} \Omega_{2}\left(I_{2}-I_{1}\right),
\end{aligned}
$$

where $I_{1}, I_{2}, I_{3}$ - are the crosspiece inertial moments relating to the axis $O x_{1}^{\prime \prime}, O x_{2}^{\prime \prime}, O x_{3}^{\prime \prime}$ respectively. Considering the equations (5) the dynamic equations of the u-joint crosspiece movement has the form:

$$
\begin{aligned}
& M_{1}=I_{1} \dot{\Omega}_{1}+\Omega_{2} \Omega_{3}\left(I_{3}-I_{2}\right), \\
& M_{2}=\left[I_{2}-\left(I_{1}-I_{3}\right) \dot{\Omega}_{2}\right], \\
& M_{3}=\left[I_{2}-\left(I_{1}-I_{3}\right) \dot{\Omega}_{2}\right] .
\end{aligned}
$$

In a specific case when $I_{3}=I_{2}$ the moments, acting on the u-joint crosspiece, are figured out with such ratios: 
The crosspiece kinetic energy is gained by:

$$
\begin{aligned}
& M_{1}=I_{1} \dot{\Omega}_{1}, \\
& M_{2}=I_{2} \dot{\Omega}_{2}, \\
& M_{3}=I_{3} \dot{\Omega}_{3} .
\end{aligned}
$$

$$
T=\frac{1}{2}\left(I_{1} \Omega_{1}^{2}+I_{2} \Omega_{2}^{2}+I_{3} \Omega_{3}^{2}\right)
$$

\section{Simulation results}

From equations (6) and (7) follows that by varying the crosspiece's inertial characteristics we can greatly change the force and energy parameters of the spinning crosspiece. In particular, from equations (6) follows that with rationing inertial moments $I_{1}=I_{3}=\frac{1}{2} I_{2}$, the external forces moment projection $M_{3}$ is zero, regardless of the angle speed of the main shaft spinning value. Figure 7 demonstrates the graphs of the force moments projection, acting on the crosspiece. Picture 8 shows the graphs of changing kinetic energy of the spinning crosspiece with different axis inertial moments ratios with the same central inertial moment $I_{O}=\frac{1}{2}\left(I_{1}+I_{2}+I_{3}\right)$, the same angle speed of the main shaft $\omega_{1}$ and the same bend angle $\alpha$.

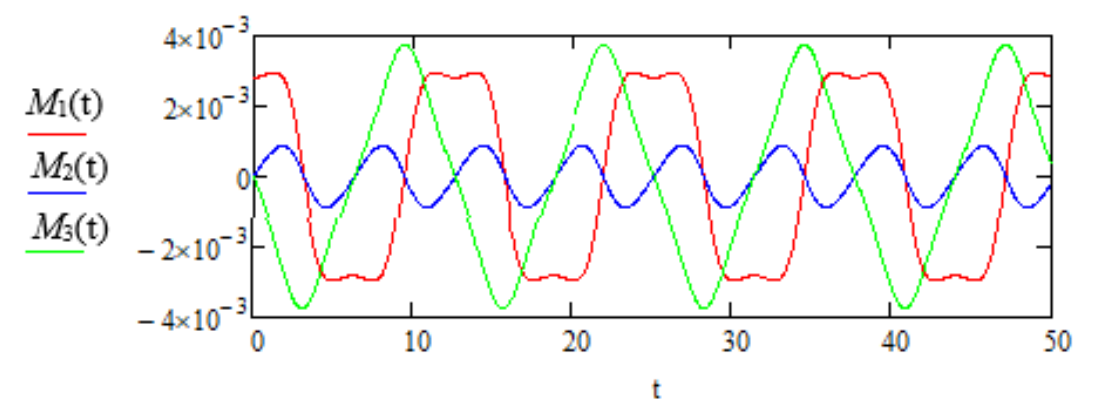

Figure 7. The dependences of force moments projection, acting on the crosspiece from time

$$
\left(\omega=0,5 \frac{\mathrm{rad}}{\mathrm{sec}}, \alpha=\frac{\pi}{6}, I_{1}=I_{2}=I_{3}=0,026 \mathrm{kgm}^{2}\right)
$$

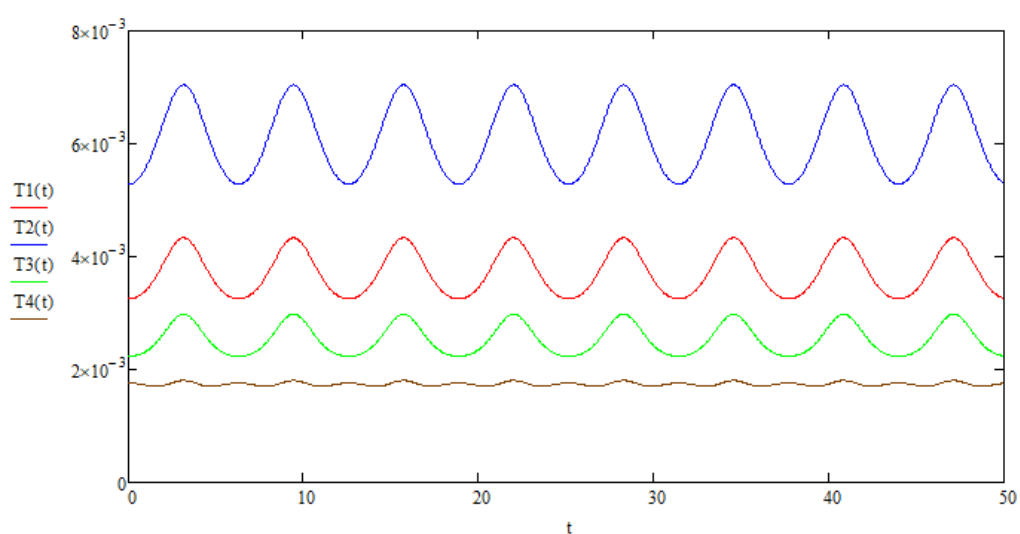

Figure 8. The dependences of changing kinetic energy $(\mathrm{J})$ from time provided

$$
\begin{aligned}
& I_{O}=0,039 \kappa г \mathrm{м}^{2} ; T_{1}: I_{1}=I_{2}=I_{3}=\frac{2}{3} I_{O} ; T_{2}: I_{1}=I_{3}=\frac{1}{3} I_{O}, \quad I_{2}=\frac{4}{3} I_{O} ; T_{3}: I_{1}=I_{3}=\frac{5}{6} I_{O}, I_{2}=\frac{1}{3} I_{O} ; \\
& T_{4}: I_{1}=\frac{7}{9} I_{O}, I_{2}=\frac{1}{9} I_{O}, I_{3}=\frac{10}{9} I_{O}
\end{aligned}
$$


As we can see on graphics on picture 8, with the constant frequency of spinning of the main shaft $\omega_{1}$ and the same bend angle $\alpha$ so the changing of the crosspiece kinetic energy is just oscillating. The vibration amplitude value significantly changes as well as an average energy. These proofs the possibility of setting up a task using the optimal mass distribution towards the spinning crosspiece.

\section{Conclusion}

In that article we examined the quaternion description of the u-joint and also found the kinematic characteristics of its movements. Studied the u-joint dynamics. With the given constant angle speed of the main shaft spinning, the angle between the axis and inertial characteristics of the crosspiece we have found its kinetic energy and force moments for u-joint plugs reactions. These acquired results may be used for creating optimal u-joints by changing its geometry.

\section{References}

[1] Cardanus H 1550 De subtilitate rerum Norimberga Devices (Johannes Petreius)

[2] Robert Hooke 1676 Description of Helioscopes, and Some Other Instruments (London: England John Martyn Electronic Materials) p 32 URL:

https://books.google.ru/books?id=KQtPAAAAcAAJ\&pg=PP5\&re - (10.02.2021)

[3] Willis R 1841 Principles of Mechanisms (London, England: John W. Parker) pp 272-284

[4] Poncelet J V 1845 Traité de mécanique appliquée aux machines (Liége: France: Librairie scientifique et industrielle) chapter 6 pp 173-214

[5] Frolov K V 2012 Theory of Mechanisms and Mechanics of Machines vol 5 (Moscow: Mechanics at a Technical University) p 686

[6] Misyura N E, Mityushov E A 2020 Quaternion models in kinematics and dynamics of spherical motion of elements of complex technical systems (Donetsk: Progressive technologies and systems of mechanical engineering) No. 4 (71) pp 27-34

[7] Golubev Y F 2013 Algebra of quaternions in rigid body kinematics (Moscow: Keldysh Institute preprints M.V. Keldysh) No. 39 p 23

[8] Animation of movement of the universal joint cross [electronic resource (Electronic Materials) URL: https://www.youtube.com/watch?v=DnEq4CxJKig. (11.02.2021) 